ations hitherto has almost equaled these figures, so that these cases which should be naturally favorable for successful operative relief, present difficulties which leave it an open question when to interfere, and how long to wait before operation is resorted to. Delay can not be said to be a safe rule in any form of intestinal obstruction. It is my belief that if all cases were operated upon within the first twenty-four hours the mortality would be reduced. We must therefore impress the general practitioners with the necessity of earlier reference of these cases to us, as we must in all cases of intestinal obstruction. We must lessen the number of last resort operations. More of the unfavorable results in operative cases are due to the condition of the patient and the parts involved, than to the fault of the operation.

The general practitioner may treat a case upon rational lines, in absence of, or because of unobtainable skilled surgical aid, and present statistics will justify him in his cours. But under what we may consider favorable surgical circumstances, I believe that fecal vomiting should be the signal for operative interference in gall-stone or any other form of obstruction, where proper and adequate measures have been tried without relief.

The measurement of this stone soon after removal was four and one-half by six inches in circumference, and was two and one-sixteenth inches in length. Marshall of Glasgow reports a case measuring one and three-quarters inches, by three and one-quarter inches in circumference. Smith and Fagge report a stone two and one-half inches, by four and one-half inches in circumferance. Conglomerate stones, or those formed by the union of several stones, have made larger bodies than single stones. Enteroliths and pure concretions become larger, but these are almost always formed in the large intestine.

Lavage of the stomach was referred to in the history, which is a treatment of great value in both the medical and surgical treatment of intestinal obstruction, in my experience. And I will here say that it is my usual practice to precede operations for the relief of intestinal obstruction with this procedure; and, while it may be the practice of many operators, I do not recall having seen, nor having heard it recommended, it having occurred to me by witnessing patients of mine almost strangle from vomitus in the larynx during anesthesia. Indeed, effort at vomiting does not have to take place to cause the stomach contents to overflow in many of these subjects, over-distended with physic and feces. The lowered head and a final palpation of the abdomen by the operator are quite sufficient to cause a flood of the fluid to embarrass the anesthetizer and endanger the patient, immediately by suffocation, and remotely from the inspiration of the highly infectious intestinal contents. The good results from this practice are always gratifying, not only during anesthesia, but following it. There was no vomiting in this case, which enabled the patient to retain salts as soon as consciousness was restored.

If the vitality of the gut is not free from suspicion it should not be returned until the proper protection has been afforded against subsequent leakage. A few Lembert sutures would have prevented the very dangerous mishap in the case reported. Omental grafts are an additional security to the line of union, and should doubtless be more frequently employed.

\section{THE USE OF ELECTRICITY BY THE GENERAL PRACTITIONER.}

Presented to the Section on Materia Medica, Pharmacy and Therapeutics, at the Forty-ninth Annual Meeting of the American Medical Association, held at Denver, Colo., June 7-10, 1898.

BY CALEB BROWN, M.D. SAC CITY, IOWA.

In the successful prosecution of any line of work it is necessary to have good tools to work with, and in no calling is this more important than in the treatment of disease, the relief of suffering and the prolongation of life, which are, or should be, the aim of every physician. Were we to examine the armamentaria of the general practitioners of medicine, we would find that where they had electrical apparatus it would, as a rule, be the farthest from up.to-date of any appliances they might have. The reasons for this are twofold: first, the market is flooded with, so far as treatment of disease is concerned, worthless but not always cheap electrical appliances, the fancied merits of these machines being constantly paraded before the medical profession by manufacturers and selling agents; second, physicians are not sufficiently well informed as to what they should or should not buy. Were we to ask every general practitioner in this country: Do you use electricity in your practice? many of them would answer yes, but should we examine their apparatus we would find in a very large per cent. of cases that their electrical outfit consisted of a so-called family or physician's battery, a box containing one or more cells, with a coil attached for the faradic current and possibly an arrangement for the galvanic current, but absolutely no reliable means of regulating the dosage. No rheostat, no milliampéremeter, none of the modern appliances.

While I do not say that such a machine is of no possible use in the treatment of disease yet I would most emphatically say that it is not what we want if we are going to do good, scientific work. As galvanism and faradism are the forms of electricity most used by the general practitioner I will confine my paper to this part of the subject, the electrocautery being used principally by the specialist, and static electricity, while it is one of the most useful forms of electric energy in the treatment of disease, yet is employed as a rule, only by those who use electricity quite extensively and are, to some extent at least, specialists in electro-therapeutics. Let us see then what apparatus is necessary for the proper use of galvanism and faradism. First is the cell for generating electric energy. Let this be a cell with carbon and zinc immersed in a solution of ammonium chlorid. For the galvanic current we will need from five to sixty or even more cells; connect these to a proper switch-board so arranged that any number of cells can be switched into circuit and also any cell or cells can be examined at any time as to their working capacity. It is then necessary to have a rheostat for controlling the current and a milliamperemeter for regulating the dose. The last named instrument is the most important, for without it no accurate work can be done, as we can only estimate the dose used by the number of cells in the circuit or the sensations of the patient, both of which are very unreliable. I will not attempt, for space will not permit, a description of a good faradic apparatus, suffice it to say we must have two to four or six cells like those in our galvanic battery, and a series of coils varying in length and fineness 
of wire, according to the condition we wish to treat, and with an interrupter that can be regulated to give slow or rapid vibrations.

A full description of the proper use of the positive and negative poles in galvanism can not be given in this paper but is better learned by a study of individual diseases in text-books on electro-therapeutics. While polarity of the faradic current has been denied by some yet there is certainly a difference in effect produced by the application of the separate electrodes. We may say as a rule that the most active polarity is the negative and should ordinarily be placed nearest the affected part. Or in general faradization the current should be directed with the blood-current.

In studying these two forms of electricity-galvan. ism and faradism-in their physiologic effects and therapeutic uses we must remember that the galvanic current is of large amperrage or volume and small voltage and its action is chemic or electrolytic, while the faradic current is electro-mechanical, having a low ampérage but a very high voltage. Having these two thoughts firmly fixed in our minds we have made a start in the right direction in the study of the effects of electric energy on the animal organism.

It we also understand the pathology of disease and have proper electric apparatus we are ready to begin the practice of electro-therapeutics; but to carry it out successfully we must understand the subject of electricity as a physical science, we must know our anatomy, physiology and pathology perfectly, we must learn to use it where indicated and dispense with it when it is contraindicated as we would any other means of combating disease. I am not of those who think electricity a panacea for every ill or that it should be universally made use of, but when intelligently used it makes our medicinal treatment more effective.

First among diseases treated by me in which I have found electricity beneficial I would place goiter. have treated this subject more fully than the limits of this paper will allow in a paper read by me last year at Harrisburg, $\mathrm{Pa}$, before the American Electro-Therapeutic Association. In that paper I gave results of treatment in quite a large number of cases of this disease. I will not devote space to describe the various forms of goiter further than to say that, aside from a true turnor growth, goiters are hard, soft, vascular or cystic, according to amount of fibrous tissue, fluids or blood-vessels entering into their structure. And we may state, in a general way, that the harder and firmer a goiter the less curative effect as to reducing its size will electricity have, yet on the other hand, although but little may be done in reducing the size of a firm goiter by electricity (aside from electropuncture), yet it will relieve to a great extent the nervous symptoms and pain, and even in exophthalmic goiter we may do much to slow the heart, calm the nervous system and improve the general condition of our patient.

The proper form of electricity in this disease is galvanism: placing one pole on the back of the neck, or behind the angle of the jaw, the other on the tumor, a very strong current is not necessary. Reverse the poles, placing first one then the other on the back of the neck or one on each side of the tumor, shifting them often enough that too great irritation of the skin will not result. Give medicine if condition of system demands it. I have never used electricity in this disease that the case was not benefited both in decrease of size of gland and relief of nervous symptoms. Many cases are cured; in some the gland has again enlarged, others have remained cured. But when we remember that latent embryonic cells may be stimulated by an enlarging thyroid body and a true tumor result, amenable to surgical treatment alone. we see the importance of curing these cases in their early stages, and for this, one of our greatest aids is undoubtedly galvanism.

We who do a general practice often have patients come to us who complain of pain in various parts of the body, muscular soreness, lame joints and a general worn-out condition. I remember seeing a statement by some medical writer that when you have a patient with a pain you can not account for in bones, joints or muscles to give iodid of potassium, I usually go one step further and use, in conjunction with the iodid or any other proper medication, general faradization, and with happy results.

In neuralgia we often have great benefit from the use of electricity. If the pain in a nerve is caused by neuritis, electricity is perhaps not our best remedy, but the further we get from the acute inflammatory condition and the nearer to the chronic inflammation or purely functional trouble the better effect we will have from this agent, and in all such cases it is indicated and is very helpful. This is markedly so in sciatica. In using faradism in this disease use a high tension induction coil with negative pole on sacrum using an electrode four to six inches in diameter; connect a smaller electrode with the positive pole and place over the course of the nerve.

To use galvanism in the same disease place a large electrode, five to nine inches in diameter, connected with negative pole over the sacrum, a somewhat smaller one connected with positive pole firmly over one of the most painful points of the nerve, increase the current from zero until a feeling of warmth is produced, hold it so for a few minutes, decrease it gradually and treat each painful point in this way. The current will need to range from five to ten, fifteen, twenty or even more milliampéres, according to the effect.

The range of diseases in which electricity may be used to advantage by the general practitioner is very wide and new uses will suggest themselves to him as his experience increases. Where I have found it most useful is in functional nerve troubles, subacute inflammations, such as chronic rheumatism, in neuralgias, lumbago-and here it is particularly usefulalso as a general tonic, and last but not least, in goiter, when used in appropriate cases.

In conclusion I would say that when we use electricity let us use it intelligently and with care, let us study it as a physical science, let us study it in its physiologic manifestations on the animal organism, but above all let us study the pathology of the diseased conditions we are to treat. Then if we will get good apparatus and use it with as much intelligence as we would give any drug, we will find electricity to be a most potent factor and a strong ally in our battle against disease.

But let us not make the mistake of considering electricity a cure-all, but ascribe to it its proper place, believing that a force that can propel cars, run the heaviest machinery, light a city or enable us to talk across miles of space, or break up molecules into their ultimate atoms, must have some effect, when properly used, upon the metabolism of the human organism. 